and his colleagues ${ }^{2}$ found that one of their patients continued to excrete Salmonella typhimurium for 17 weeks from the time of getting the infection. They calculated that the decline in the population of excreters followed almost exactly the pattern which had been reported previously in an outbreak of infection with Salmonella paratyphi-B described by T. C. R. George, R. W. S. Harvey, and S. Thomson. ${ }^{3}$ The data presented by Semple and his colleagues are not in such a form as to permit comparison with those reported by these other workers, but it would be worth knowing whether the decline in the number of excreters after an outbreak of salmonellosis follows a recognizable and predictable pattern.

The outbreak of human food-poisoning prompted bacteriological investigations into the packing station and 14 chickenrearing farms in Cheshire from which the poultry which had caused the outbreak were supplied. J. H. Pennington and his colleagues report at page 804 that $S$. virchow was isolated in nine farms, the packing station, and the hatchery, while several of the workers on the farms were infected. Cattle grazing on land manured with litter from two of the farms, one of which was infected, themselves became infected with $S$. virchow.

One of the factors responsible for disseminating the infection even more widely to other foodstuffs was poor hygiene in the kitchen of the premises in which the chicken was prepared. It is clear from the account given by Semple and his colleagues that the working place was inadequate and the preparation of the food unhygienic. Moreover, the infected chicken had been deep-frozen and was spit-roasted after a very brief thawing period, so that it is unlikely that the cooking can have raised the temperature of the carcasses high enough to have significantly reduced the population of the pathogens in and on them. All too often food is prepared in catering establishments in Britain under thoroughly unsuitable conditions. It is not at all easy to understand why the public health authorities seem so reluctant to use the formidable powers devolved upon them by the Ministry of Agriculture and the Department of Health and Social Security. Under the Food and Drugs Act, 1955, they can insist on a hygienic environment and a healthy work force in the catering industry. After all, to keep the lid of Pandora's box firmly closed is the true purpose of preventive medicine.

\section{International Co-operation}

In the last few years substantial technical developments have been made in the control of tropical disease. Mass campaigns and new methods have restricted the extent of yaws, poliomyelitis, tuberculosis, and yellow fever. Malaria eradication programmes have been successful over wide areas. Advances have been made in chemotherapy and chemoprophylaxis, and new or better vaccines have been produced.

Despite these developments, however, there is still the threat of widespread outbreaks of some of the quarantinable diseases. For example, cholera el $\operatorname{Tor}^{12}$ has recently spread from Sukuwesi (Celebes) to New Guinea, the Philippines, China, Hong Kong, Thailand, Southern Russia, Iran, and Iraq. At p. 789 of this week's B.M.7. Dr. P. Dorolle, the Deputy Director of the World Health Organization, argues that the existing international sanitary regulations are inadequate. Some countries have introduced exaggerated precautions much in excess of those prescribed by the inter- national regulations. Others have deliberately failed to notify an outbreak because of the fear of excessive reactions from other countries. Moreover, the current regulations have failed in other ways, notably in preventing the introduction of smallpox into non-epidemic countries. Dr. Dorolle concludes that the regulations can work only when they are properly applied in a co-operative effort. They have failed from lack of accurate reporting, failure of detection, and difficulty of enforcement.

Clearly, therefore, in an age of great and increasing speed of travel and increasing numbers of international travellers the old regulations are inadequate. This situation was discussed at a conference on international co-operation held in 1967 by W.H.O. An important outcome of this and other discussions on the quarantinable diseases was the demand for a complete review of the international sanitary regulations. This was recommended at the 21st World Health Assembly in May this year, and, in keeping with this proposal, W.H.O. has merged its old division of International Quarantine with that of Global Epidemiological Surveillance.

Hence the regulations should soon be given a new and modern look, but until then they will have to be enforcedwe hope with more understanding. In the past they have been negative and compulsive, and have been carried out with a "legalistic and regulation-minded" attitude. Dr. Dorolle voices the views of the vast majority of those who work in this field when he hopes that this rigid philosophy will give way nationally and internationally to a more scientific and technical approach. The latter should be based on the introduction of epidemiological surveillance, amounting to continuous scrutiny of the distribution and spread of communicable diseases, the prediction of the development of potentially serious situations, and the collection of data of sufficient accuracy and completeness to serve as a basis for effective control.

What can be done in this field has been shown by the growth of the international surveillance of influenza, which is not one of the "quarantinable" diseases, though from time to time it causes world pandemics. A World Influenza Centre was established in the Medical Research Council Laboratories in London in 1947 ; today there are over 50 countries collaboratin. $n$ a world programme, collecting and distributing information on the national and global occurrence of the disease and on the details of the viruses concerned and their antigenic structure, with a view to the ultimate production of vaccine. The programme is based on voluntary collaboration on a scientific and professional level with no element of compulsion. It is a good example of the way in which detection and notification of a communicable disease beginning at national level can be extended to co-operative international surveillance.

Such an approach will do much to prevent the spread of disease, but the real solution lies ultimately in eliminating the endemic foci altogether-though the examples of diseases like malaria and smallpox, of which areas of infection persist despite a careful and thorough attack, show how difficult this will be.

With few exceptions, the major diseases concerned are those which originate from countries which are at present underdeveloped and in which the traditional methods of control, such as repeated mass vaccination in smallpox, are too difficult to deploy and the newer surveillance too dependent on technical staff and labour which do not exist. In

\footnotetext{
Brit. med. 7., 1966, 1, 1555.

Wallace, C. K., et al., Brit. med. F., 1966, 2, 447
} 
the end the problem is largely an economic one, and, as Dr. Dorolle rightly says, "the only way of preventing the old plagues, and some new ones, from spreading from continent to continent and from country to country is to help the poorest nations of the world to reach such a level of economic and technical development that it will be possible for them to combat the evil at source."

\section{Therapeutic Abortion}

Recent changes in the law have resulted in an increase in the number of patients submitted for therapeutic abortion. It is opportune therefore to consider the methods available for terminating pregnancy and their limitations and dangers.

In the first three months of pregnancy the quickest method of termination is by dilatation of the cervix with graduated dilators and evacuation of the uterus with finger and ring (or ovum) forceps. But emptying the uterus of its contents is not always an easy matter even for the skilled gynaecologist, and there are certainly many pitfalls for the inexperienced practitioner. Unless great care is taken in dilatation the cervix may be split longitudinally. The slow introduction of each dilator, pausing briefly before withdrawing it and proceeding to the next, reduces this danger and also helps promote uterine contraction. The pregnant uterus is soft, so that dilators may easily perforate its anterior wall if retroversion is unrecognized or the posterior wall if the uterus is, wrongly, thought to be retroverted. An intravenous injection of ergometrine given before dilating the cervix will help mitigate the danger of bleeding, while if bleeding is troublesome a blunt flushing curette using hot saline $-118^{\circ} \mathrm{F} .\left(48^{\circ} \mathrm{C}\right.$.) - has a haemostatic effect. But haemorrhage can be alarming, and most gynaecologists have had an unnerving experience when evacuating a pregnant uterus. The difficulties increase as pregnancy advances-so much so that after the 12 th or certainly after the 14 th week (the actual size of the uterus is more critical than the duration of amenorrhoea) evacuation in one stage is best avoided.

If abortion by the vaginal route is nevertheless favoured and if there is no urgency, the risks of forcible dilatation of the cervix can be avoided by the insertion of one or more laminaria tents. As the tents swell the cervix is slowly dilated to permit evacuation of the uterine contents 24 hours later. The risk of mishap is slight, but this method-familiar to an older generation of gynaecologists-is nowadays comparatively little known and not much favoured. Nor does it solve all the problems.

Another possible alternative to the one-stage operation is the injection of an abortifacient paste between the membranes and the uterine wall. This procedure will usually be followed by expulsion of the ovum and often it is complete, though in a minority of cases abortion may be incomplete and subsequent evacuation necessary. This simple method usually works well and has been widely used, but unfortunately it is not free from risk, and sudden death from embolism or vagal inhibition has been reported.

If the uterus is enlarged to more than the size of a 14-weeks pregnancy and is palpable abdominally, most gynaecologists prefer to perform an abdominal hysterotomy. This is, in effect, a caesarean section in miniature. The operation can conveniently be performed through a Pfannenstiel incision. An oxytocic injection given before opening the uterus mini- mizes haemorrhage and promotes uterine contraction and retraction. A low vertical incision into the uterus is usually preferred and if the bladder peritoneum is first reflected downwards it can be used subsequently to cover the incision, which is sutured in two layers. The ovum can usually be extracted intact, decidua swabbed away, and the uterus seen to be emptied completely.

In skilled hands abdominal hysterotomy is a safe procedure, and it has the advantage that it can be combined with sterilization if desired. Its one drawback is that occasionally decidual cells may be implanted in the abdominal wall or pelvic peritoneum, with subsequent development of endometriosis. The risks which once attended abdominal surgery have so diminished that nowadays there would seem to be no indication for performing vaginal hysterotomy.

Apart from these traditional methods a number of new techniques have been suggested in recent years. Among these is the intrauterine injection of hypertonic saline or glucose. This method is applicable particularly when pregnancy has advanced beyond the 12th week or in cases of intrauterine death. With a suitable long needle as much amniotic fluid as possible is removed and replaced with a corresponding volume of up to $200 \mathrm{ml}$. of $20 \%$ saline $^{2}$ or $50 \%$ glucose. ${ }^{3}$ The needle is usually inserted through the abdominal wall, but the vaginal route may be favoured. If there is difficulty in locating the amniotic sac-and the smaller the uterus the more difficult it is -an alternative is to inject saline into the extra-amniotic space via a Foley catheter in the cervix. In most cases expulsion of the uterine contents occurs 2448 hours after injection.

Though some authors have commended the safety of the method, ${ }^{3}$ there is a risk of infection after the injection of glucose. Anaerobic organisms-including Clostridium welchii-can flourish in a glucose medium, and serious sepsis has been reported. For this reason many gynaecologists have preferred saline. Recently, however, there have been disquieting reports of several deaths ${ }^{4}$ (characterized by focal intracranial haemorrhage) following the injection of saline. These are tragic mishaps, and unless suggested safeguards ${ }^{5}$ to avoid the risks of intravascular injection and hypernatraemia can ensure the safety of the method it cannot continue to be recommended. In this connexion it is worth noting that though it was once widely used in Japan it has been largely given up. ${ }^{6}$

Vacuum suction has been recently introduced and provides a promising (and possibly safer) alternative to the conventional method of evacuating the uterus in the first 12-14 weeks of pregnancy. It appears to have been first described in China in 1958, and there are now many reports of its use on many thousands of patients without serious complication. ${ }^{78}$ Recently it has been adopted in Britain. The cervix is first dilated. A hollow suction tube of convenient size is selected and introduced in the uterus. A negative pressure of $0.4-0.6 \mathrm{~kg} . /$ sq. $\mathrm{cm}$. is produced, and, with the suction tube used rather like a curette, the conception products are broken up and withdrawn into a suction bottle. The procedure is technically simple, and with a little practice the uterine contents can be aspirated cleanly and quickly. It is claimed that less dilatation

\footnotetext{
1 Wood, C., Booth, R. T., and Pinkerton, J. H. M., Brit. med. F., 1962,

${ }_{2}^{2,706 .}$ Bengtsson, L. P., and Csapo, A., Amer. F. Obstet. Gynec., 1962, 83, 1083.

3 Brosset, A., Acta obstet. gynec. scand., 1954, 33, 445.

- Cameron, J. M., and Dayan, A. D., Brit. med f., 1966, 1, 1010

- Fuchs, F., quoted in Obstet. Gynec. Surv., 1966, 21, 739.

- Wagatsuma, T., Amer. F. Obstet. Gynec., 1965, 93, 743.

Chalupa, M., Z̈bl. Gynäk., 1964, 86. 1803.

- Nilsson, C.-A., Acta. obster. gynec. scand., 1967, 46, 501.
} 